was violent and explosive. The peak prevalence was at school entry, and specific learning difficulties were common. They blamed injury or dysfunction of the diencephalon-despite claiming recovery between the ages of 8 and 18 years. The frustration felt by intelligent children who are school failures might sometimes have been the explanation.

Over the years the syndrome has been modified: the elements surrounding the central "excessive" motor activity have increased, and the diagnosis is now made in the United States in half of all children referred to psychiatrists. In contrast, British practice generally limits the diagnosis to children with epilepsy, low IQ, and recognisable neurological problems-a difference that has led Rutter ${ }^{4}$ to seek an explanation.

In Rutter's own classification the American description fitted a conduct disorder. Variables common to both diagnoses include male predominance; complications of pregnancy and perinatal morbidity (usually accepted on the mother's word without hospital confirmation); physical anomalies; learning disorders; attention deficits; poor prognosis; and "sociopathy" and antisocial disorders in parents. Rutter questioned whether a broadly based diagnosis of "hyperkinetic syndrome" differentiates it "from other psychiatric disorders in terms of aetiology, course, response to treatment, or some other clinical variable." In the event he did not test whether the hyperkinetic syndrome fulfilled his own postulates for a recognisable entity. Instead, he and his colleagues ${ }^{5}$ asked a different question. Given a diagnosis of a conduct disorder, does the subdivision into those boys with and those without associated hyperkinesis have any validity? To this question the general answer was no.

The sample comprised 68 boys aged 5 to 11 years referred for psychiatric assessment or treatment to the Maudsley Hospital or the Brixton Child Guidance Centre during one year; it excluded psychotic children and any with IQ under 50. The methods used (and fully described) included, among a broad range of tests, scored questionnaires filled in by teachers and parents and observational measures of activity and attention. With hyperactivity as the symptom-in-chief the finding is important that there was no significant correlation between parents' and teachers' hyperactivity scores or between these and the observational scores. In individual cases overactive behaviour seemed to be found in some specific circumstances but not others-an observation also made by Campbell ${ }^{6}$ in Montreal, who differentiated "situation hyperactivity" from true hyperactivity, which is "cross-situational." Rutter found few differences between "psychiatrically abnormal children with or without hyperkinesis when compared on cognitive functioning, perinatal history, neurological examination, congenital abnormalities, and psychosocial circumstances." $\mathrm{He}$ delineated a clinical picture of generalised overactivity with neurological immaturities, an erratic style of cognitive functioning, and an early onset, which might, perhaps, be a distinct entity; but this was rare.

Nevertheless, diagnoses which can never reflect the whole truth about patients may prove valuable as concepts. Is the "hyperkinetic syndrome" a valid diagnosis? The answer depends not only on careful statistical correlations but on the use made of diagnostic labels. The medical model for defining diagnosis is, unfortunately, based on bacterial infections with distinctive symptoms and signs, predictable course and outcome, and a relatively consistent response to treatment-and all backed up with positive cultures. Is this model appropriate to the study of disturbances of conduct and behaviour and reaction to stress - or even of the kinds of disease that underlie "soft" neurological signs? Historically, many of our now respectable diagnoses started life as labels. Over 100 entries under the rubric of hyperkinesis appeared in both the 1976 and the 1977 volumes of the Cumulative Index Medicus. Despite Rutter's intellectual dissatisfaction the hyperkinetic syndrome seems likely to remain on the list.

${ }^{1}$ Bradley, C, American fournal of Psychiatry, 1938, 94, 577.

2 Ounsted, C, Lancet, 1955, 2, 303.

${ }^{3}$ Laufer, M W, and Denhoff, E, Fournal of Pediatrics, 1957, 50, 463.

4 Rutter, M, fournal of Child Psychology and Psychiatry, 1965, 6, 71.

5 Sandberg, S T, Rutter, M, and Taylor, E, Developmental Medicine and Child Neurology, 1978, 20, 279.

- Campbell, S B, Endman, M W, and Bernfeld, G, fournal of Child Psychology and Psychiatry, 1977, 18, 239.

\section{The physicians' dilemma}

\begin{abstract}
"In the training centre the vast majority of the physicians are specialists; in the real world the vast majority of the care needed is general. In the academic centre the pressure is to carry out detailed scientific evaluation, interpret physiologic data in relation to disease, and to act in the short-term-in the real world the need is for understanding of the illness engendered by the disease and its effects on the patient and his family, and for long-term action tempered by caution and care."'
\end{abstract}

A series of papers on the specialist versus generalist debate has recently been occupying the columns of the New England fournal of Medicine. The increasing financial cost of medical manpower in the United States puts an onus on the medical profession to produce the right kind of doctor in the right place. Yet for some time now there has been increasing emphasis on the training of medical subspecialists rather than of internists (general physicians), and these tend to concentrate in the prestigious medical centres. Internal medicine is the most popular choice for residency training there, with 15000 trainees in any one year, but $70^{\circ}$ of those who complete their residencies go on to subspecialty training. ${ }^{2}$ There are many reasons for this. The staff of departments of internal medicine is largely made up of subspecialists, who need trainees to help with procedures such as cardiac catheterisation and endoscopy, as well as research. It seems easier to get funds to support the subspecialties, and both the department and its fellows benefit from the prestige of such grants. Board certification can be obtained more quickly if the trainee does one year in a subspecialty, and he can then look forward to earning more money for less work, especially if he is skilled in particular techniques, than his fellow internists. Yet paradoxically most trainees are destined to practise general internal medicine (primary care) for much of their time, and only a minority will be whole-time practitioners of one of the 10 subspecialties certifiable by the American Board of Internal Medicine.

Various suggestions have been made to halt what is thought to be an increasing and unwelcome trend towards specialisation. The spectacular success in the past 10 years of family practice training schemes, which now number some 350 with over 5000 residents in training, ${ }^{3}$ has stimulated interest in a similar type of training in primary care for hospital physicians. Any such scheme would undoubtedly require reassessment of present certification procedures in internal medicine, because the emphasis would be on experience in the wards and clinics rather than on research and laboratory work, and it has 
even been suggested that subjects such as outpatient gynaecology and orthopaedics should be included, ${ }^{3}$ as well as those more traditionally associated with general medicine such as paediatrics and psychiatry. The aim would be at producing a hospital internist capable of caring for people "from the neonatal period to the geriatric age group." ${ }^{1} \mathrm{He}$ could perhaps be produced by a co-operative training programme by departments of medicine, paediatrics, family practice, and others; a single primary care residency programme with three tracks leading to certification in either internal medicine, paediatrics, or family practice is already in operation at Montefiore Hospital in New York. ${ }^{4}$

The main danger of such programmes seems to be in feeding the internist too much information. Suggestions, for example, that there should be greater emphasis on social and behavioural sciences should surely be referred to the undergraduate curriculum, and the ambition to produce a wellrounded, caring internist should not be allowed to obscure the fact that a good doctor goes on learning. Besides, in the fullness of time it will surely be accepted that some parts of an internist's work can be carried out by health professionals. A valuable lesson that emerged from family practice programmes $^{3}$ was that the doctor's functional role was more important than the extent of his skills and knowledge.

By no means everyone agrees that increasing specialisation in medicine is necessarily bad, or indeed that there is a crisis in the United States which needs desperate remedies. ${ }^{56} \mathrm{Re}$ assurance comes from those who point out that on average specialists spend half their time in primary care and there is no evidence that they are worse at it than internists. The danger of producing over-trained, academic physicians, moreover, may have been exaggerated, since three-quarters of the residencies in general internal medicine are in community hospitals. ${ }^{7}$ Furthermore, academics argue $^{6}$ that it is irresponsible to destroy programmes in the subspecialties, which are often the source of new knowledge, for reasons of political expediency.

Here in Britain doubts have also been raised about the wisdom of increasing specialisation within medicine. The growth of excellent rotating training programmes has meant that the young physician is not only exposed early to the subspecialties but also obtains the MRCP after perhaps less than two years spent in general medicine. He is then likely to find himself having to apply for appointments in the subspecialties. In a recent (unpublished) analysis of advertisements D N S Kerr found that $60^{\circ}{ }_{0}$ of those for consultant posts asked for a special interest and nearly a quarter more stated that it would be an advantage. This means that under a quarter of all new consultants are expected to be solely general physicians. Should a small country like Britain, with an impoverished health service, expect every district hospital to have a full complement of subspecialists ? The colleges (and not only in medicine) have encouraged specialisation by a justifiable desire to formulate proper training programmes; this has led to a certain rigidity not only in the training itself but also in the expectations of those responsible for making appointments.

It may be true that as health education improves sick people will increasingly demand specialist advice, but there is a danger of fragmentation of medical care if no one takes overall responsibility. The general physician should still have a future as the patient's "friend." Bernard Shaw (and Beveridge) thought that the manpower needs of a comprehensive health service were predictable, and that once we got the equation right we would all become healthier. No one would argue that complex problems are best dealt with by one or more specialists, but should the patient with a common, uncomplicated illness such as a duodenal ulcer be treated by a general physician (internist) or by a specialist gastroenterologist?

${ }^{1}$ Perkoff, G T, New England fournal of Medicine, 1978, 299, 654

2 Petersdorf, R G, New England fournal of Medicine, 1978, 299, 628.

${ }^{3}$ Colwill, J M, New England fournal of Medicine, 1978, 299, 657.

+ Boufford, J I, Annals of Internal Medicine, 1977, 87, 359.

${ }^{5}$ Reitmeier, R J, and Benson, J A, New England fournal of Medicine, 1978, 299, 1308.

6 Thier, S O, and Berliner, R W, New England fournal of Medicine, 1978, 299, 1305.

' Eisenberg, J M, New England fournal of Medicine, 1978, 299, 1309.

\section{Proteinuria at high altitude}

The expansion of high-altitude trekking, especially in the Himalayas, has led to increased awareness of the medical problems of high altitude. The symptoms of mountain sickness and pulmonary and cerebral oedema may be dramatic and these conditions have been extensively studied both in laboratory conditions and on the mountains. Retinal haemorrhage may, however, pass unnoticed (unless it affects the macula); high-altitude proteinuria is symptomless and no hazard in itself and has attracted even less attention. Neither Heath and Williams's book Man at High Altitude nor the Alpine Club's Mountain Medicine and Physiology deals with proteinuria, nor did Singh discuss it in his account of his extensive experience with the medical problems of altitude experienced by the Indian Army in 1968. ${ }^{1}$

Proteinuria has now been studied at high altitude by Arnold Pines, who continues the tradition of the British doctor physiologist mountaineer. $\mathrm{He}$ observed $^{2}$ seven volunteers who spent six weeks in the East African mountains, during which time they made five separate climbs. On the first two climbs the mean urine protein concentration in morning specimens ranged from 30 to $300 \mathrm{mg} 100 \mathrm{ml}$, but on the last three it had dropped to around $15 \mathrm{mg} 100 \mathrm{ml}$-an arbitrary figure assigned to the "trace" colour on Bili-Labstix. The highest protein concentrations were found in climbers who had symptoms or signs of mountain sickness. Since proteinuria did not occur during strenuous exercise at medium altitude it was probably a response to height rather than exertion, and its diminution over a period of weeks indicated that the kidneys share in acclimatisation.

Rennie and Joseph ${ }^{3}$ had earlier shown that there was a correlation between altitude and mean urine protein concentration; that the time lag in its development after ascent was similar to that found in mountain sickness; and that there was considerable person-to-person variation. Rennie et $a l^{4}$ also found that the urine protein concentration in young adult males of Quechua descent, born and living at high altitude in the Andes, was greater than that in their compatriots who had moved down near sea level. They drew a parallel with the proteinuria found in sufferers from cyanotic congenital heart disease.

Climbers have long been aware that ascent to high altitude may be associated with changes in urine volume. Oliguria points to an increased likelihood of the development of mountain sickness, while high-altitude diuresis suggests that this is unlikely to occur. Aldosterone secretion falls, especially in sufferers from mountain sickness. Urine sodium excretion 